\title{
Generalized Dobrushin Coefficients on Banach Spaces
}

\section{Wojciech Bartoszek ${ }^{1,2}$ (D) Marek Beśka ${ }^{2}$. Wiktor Florek ${ }^{1,2}$}

Received: 17 October 2020 / Revised: 14 May 2021 / Accepted: 8 June 2021 /

Published online: 18 July 2021

(c) The Author(s) 2021

\section{Abstract}

The asymptotic behavior of iterates of bounded linear operators (not necessarily positive), acting on Banach spaces, is studied. Through the Dobrushin ergodicity coefficient, we generalize some ergodic theorems obtained earlier for classical Markov semigroups acting on $L^{1}$ (or positive operators on abstract state spaces).

Keywords Dobrushin coefficient · Markov operator · Mixing · Category

Mathematics Subject Classification 47A35 $\cdot 37$ A30 $\cdot$ 60J05

\section{Introduction}

Classical results on asymptotic behavior of iterates of Markov operators (or oneparameter semigroups) have been recently generalized in different directions. In most cases, domains like Banach lattices are replaced by abstract ordered Banach spaces. To this end, the concept of a Markov operator is properly adjusted, and necessary geometric features are imposed. In this paper, we further contribute to these extensions. More specifically, our main goal is to modify Dobrushin methods for new abstract domains. We furthermore simplify existing proofs. Most results from [27,28] are formulated in terms of the so-called abstract state spaces $\left(\mathfrak{X}, \mathfrak{X}_{+}, \mathcal{K}, f\right)$, special classes

Communicated by Gholam H. Esslamzadeh.

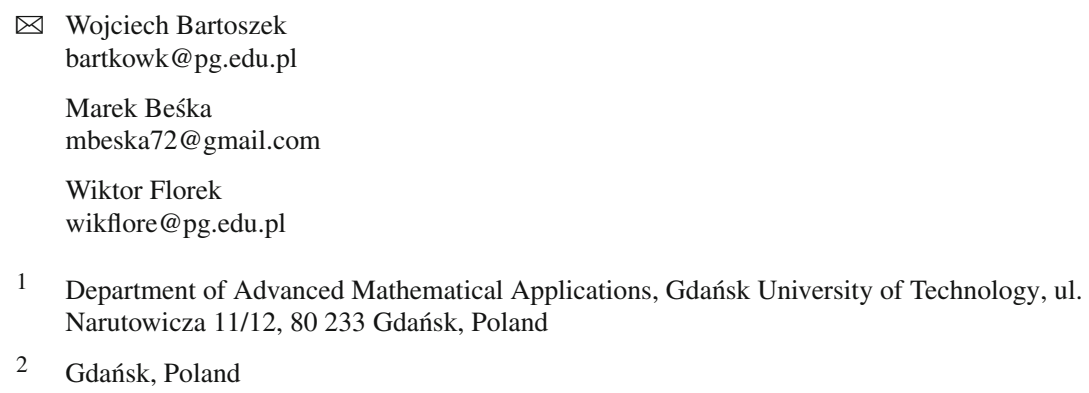


of ordered Banach spaces, where the norms are additive on positive cones, $\mathfrak{X}_{+}$, and the considered operators are usually Markovian. We shall also introduce corresponding (imitating Markov properties) class of operators; however, the additivity of the norm will not be a prerequisite.

The Dobrushin (Dobrushin-Doeblin actually) ergodicity coefficient was introduced in 1955 (cf. [12,33]) From a general perspective, it appeared to be an efficient tool for studying the asymptotic behavior of Markov chains and their limits. Moreover, it allows for obtaining the rate of convergence. When a stochastic system is defined in the language of operator theory, then the evolution of a Markov process $\left\{\xi_{t}\right\}_{t \in \Theta}$ can be seen as iterates $T^{n}$ of the related transition operator $T$. In an elementary course on Markov chains, where the phase spaces are finite, $T$ may be identified with a transition (raw stochastic) matrix $T=\left[p_{j, k}\right]_{n \times n}, \sum_{k=1}^{n} p_{j, k}=1$ for all $1 \leq j \leq n$ and $p_{j, k} \geq 0 . T$ acts on the (Banach) space $\ell_{n}^{1}=\mathbb{R}^{n}$ as $T(\underline{x})=$ $\left(x_{1}, \ldots, x_{n}\right) \circ\left[p_{j, k}\right]=\left(\sum_{j=1}^{n} x_{j} p_{j, k}\right)_{k=1 \ldots n}$. Generalizing $\left[p_{j, k}\right]_{n \times n}$ to a system of transition probabilities $\{P(x, A): x \in G, A \in \mathcal{G}\}$, where $(G, \mathcal{G})$ is a fixed measurable space (called a phase space), we identify Markov processes $\left\{X_{n}\right\}_{n \geq 0}$ on $G$ with linear operators $T: \mathcal{M}(G, \mathcal{G}) \rightarrow \mathcal{M}(G, \mathcal{G})$ defined as $T(\nu)(A)=\int_{G} P(x, A) d \nu(x)$, where $\mathcal{M}(G, \mathcal{G})$ stands for the Banach lattice of finite $\sigma$-additive signed measures on $(G, \mathcal{G})$. If for all (sometimes, it is required $\mu$ almost all) $x \in G$, the probability measures $P(x, \cdot) \prec \mu$ are absolutely continuous with respect to $\mu$, then $T: L^{1}(\mu) \rightarrow L^{1}(\mu)$. The limit (if it exists) of $T^{n}(\nu)$ is a stationary distribution, depending on an initial distribution $v=\mathcal{L}\left(X_{0}\right)$. A common feature of these examples is the fact that $T$ is a positive linear contraction acting on a (real) Banach lattice. Using functional analysis language, Markov processes emerge as linear and positive operators $T$ (called Markov or stochastic), which are defined on some function Banach space $\mathfrak{X}\left(=L^{p}\right.$ or $\left.C(S)\right)$. Of course, these Banach spaces $\mathfrak{X}$ and operators $T: \mathfrak{X} \rightarrow \mathfrak{X}$ must be carefully chosen for appropriate modeling of a specific random phenomenon.

Apart from the Dobrushin method, there are other techniques used in studying the convergence of iterates of positive operators. The method of lower and upper bounds, introduced by Lasota (cf. [22]), is very popular, but it can also be interpreted in Dobrushin terminology. In the classical situation, when $\mathfrak{X}=\ell_{n}^{1}$, the Dobrushin ergodicity coefficient of a linear Markov operator $T: \ell_{n}^{1} \rightarrow \ell_{n}^{1}$ is defined as

$$
(\star) \quad \delta(T)=\sup \left\{\frac{\|T(\mu)-T(v)\|_{1}}{\|\mu-v\|_{1}}: \mu \neq v \in \mathcal{D}_{n}\right\},
$$

where $\mathcal{D}_{n}=\left\{\left(x_{1}, \ldots, x_{n}\right): \sum_{j=1}^{n} x_{j}=1\right.$, and $\left.x_{1}, \ldots, x_{n} \geq 0\right\}$ denotes the simplex of probability vectors and $\|\cdot\|_{1}$ stands for the $\ell_{n}^{1}$ norm.

The methods and techniques of functional analysis are especially suited for studying noncommutative Markov processes (whose theory is still being developed ([9,13, 34]). For example, the mathematics behind quantum theories require mathematical objects, which are not Banach lattices in general. Here, the states are not probability densities, but are (positive, norm 1, normal) functionals on Von Neumann algebras. These are not Riesz spaces, and therefore, the evolution operators $T$ are not defined on Banach lattices any more. Nevertheless, all $C^{*}$-algebras have natural order structures, compatible with linear operations. Positive (very often completely positive) operators 
$T$ describing quantum evolutions act on ordered Banach spaces (cf. [1,2,5,6,10,11,29, $30,35])$. The topic of positive linear operators on ordered Banach spaces, which are not necessarily Banach lattices, has attracted significant attention (e.g., [8,14-17,2428,32])

In the first section of the paper, we present basic general results, where the principal domains are abstract (real) Banach spaces $(\mathfrak{X},\|\cdot\|)$. The Banach algebra of all bounded linear operators $T: \mathfrak{X} \rightarrow \mathfrak{X}$ is denoted by $\mathcal{L}(\mathfrak{X})$. The operator norm $\|T\|$ is traditionally denoted by $\|\cdot\|$ as well.

\section{Abstract Dobrushin Coefficient}

Following [27, Definition 3.1], we introduce the following.

Definition 2.1 Let $T \in \mathcal{L}(\mathfrak{X})$ be a linear operator and $P \in \mathcal{L}(\mathfrak{X})$ be a (nontrivial) linear projection. Then, the following quantity:

$$
\delta_{P}(T)=\sup _{x \in N_{P}, x \neq 0} \frac{\|T x\|}{\|x\|},
$$

where $N_{P}=\{y \in \mathfrak{X}: P y=0\}$, is called the Dobrushin coefficient of $T$ with respect to $P$ (if $N_{P}=\{0\}$, i.e., when $P=I$, we may artificially prescribe $\delta_{P}(T)=1$ ).

Clearly, $0 \leq \delta_{P}(T) \leq\|T\|$. We start with a commonly known result on the Dobrushin coefficient. For its proof, the reader is directed to [27, Theorem 3.7].

Theorem 2.2 Let $T, S \in \mathcal{L}(\mathfrak{X})$ and $P, Q: \mathfrak{X} \rightarrow \mathfrak{X}$ be linear projections. Then

(1) if a projection $P$ dominates a projection $Q$ (i.e., $Q P=P Q=Q)$, then $\delta_{P}(T) \leq$ $\delta_{Q}(T)$,

(2) $\left|\delta_{P}(T)-\delta_{P}(S)\right| \leq \delta_{P}(T-S) \leq\|T-S\|$,

(3) $\delta_{P}(T+S) \leq \delta_{P}(T)+\delta_{P}(S)$,

(4) $\delta_{P}(T S) \leq\|T\| \delta_{P}(S)$,

(5) $\|T S\| \leq \delta_{P}(T)\|S\|$, if $P S=0$,

(6) $\delta_{P}(T S) \leq \delta_{P}(T) \delta_{P}(S) \leq \delta_{P}(T)\|S\|$, if $S\left(N_{P}\right) \subseteq N_{P}$.

The following result is also a standard fact and holds for abstract linear operators on Banach spaces.

Proposition 2.3 Let $T \in \mathcal{L}(\mathfrak{X})$ and $P: \mathfrak{X} \rightarrow \mathfrak{X}$ be a linear projection. If $\delta_{P}(T)=0$, then $T=T P$.

Proof If $x \in N_{P}$, then $0=T x=T x-T P x$, so $T=T P$ on $N_{P}$. Generally, if $y \in \mathfrak{X}$, then $y-P y \in N_{P}$, so $T(y-P y)=T P(y-P y)=0$. We get $T y=T P y$.

The next result will play an essential role in the sequel. In [27], most of the results, on asymptotic behavior of iterates $T^{n}$, were obtained under additional assumption that $P T=T P$, i.e., that $T$ commutes with $P$. In this paper, we relax this condition, 
which is replaced by essentially weaker $T\left(N_{P}\right) \subseteq N_{P}$. Recently, a similar remark was pointed out in [28, Lemma 2.7], where the assumption $P T=P T P$ is in use. Indeed, commutativity implies that for all $x \in N_{P}$, we have $P T x=T P x=T 0=0$. We also notice that if $T\left(N_{P}\right) \subseteq N_{P}$ and $T P=P$, then $T P=P T=P$. In fact, $P T=P T(I-P+P)=P T(I-P)+P=P$ as $(I-P) \mathfrak{X}=N_{P}$.

Proposition 2.4 Let $T \in \mathcal{L}(\mathfrak{X})$ and $P: \mathfrak{X} \rightarrow \mathfrak{X}$ be a linear projection. If $\delta_{P}(T)<1$ and $T\left(N_{P}\right) \subseteq N_{P}$, then $\left\|T^{n}(I-P)\right\| \rightarrow 0$.

Proof The inequality $\delta_{P}(T)<1$ simply means that the operator $T_{N_{P}}=T \uparrow_{N_{P}}$, the restriction of $T$ to its invariant subspace $N_{P}$, is a strict contraction. It follows that $\left\|T_{N_{P}}^{n}\right\| \leq\left(\delta_{P}(T)\right)^{n} \rightarrow 0$. In particular, $\left\|T^{n}(I-P)\right\| \rightarrow 0$ as $(I-P)(\mathfrak{X})=N_{P}$.

Let us present an example which shows that $T P=P T=P$ is essentially stronger than $T\left(N_{P}\right) \subseteq N_{P}$, even in classical situations.

Example 2.5 Let $\mathfrak{X}=L^{1}(\mu)$, where $\mu$ is a $\sigma$-finite positive measure on a measurable space $(G, \mathcal{G})$. By $\mathcal{D}$, we denote the convex set of densities, i.e., $g \in \mathcal{D}$ if and only if $g \geq 0, \mu$ a.e. and $\int_{G} g d \mu=1$. For a fixed $g_{*} \in \mathcal{D}$, define a linear projection $P f=\left(\int_{G} f d \mu\right) g_{*}$, i.e., $P=\mathbf{1} \otimes g_{*}$. Let $T$ be a general Markovian operator on $L^{1}(\mu)$, i.e., $T f \geq 0$ for all $f \geq 0$ and $T^{*} \mathbf{1}=\mathbf{1}$, where $T^{*}$ stands for the adjoint operator $T^{*}: L^{\infty}(\mu) \rightarrow L^{\infty}(\mu)$. It is easily seen that $P T f=P f$ for all $f \in L^{1}(\mu)$, but $T P f=P f$ if and only if $T g_{*}=g_{*}$. Thus, $P$ and $T$ do not commute in general. However, $f \in N_{P}$ if and only if $\int_{\Omega} f d \mu=0$. For such $f$, we have $\int_{G} T f d \mu=0$ as well, so $T\left(N_{P}\right) \subseteq N_{P}$.

In the next theorem, we generalize [27, Theorem 3.14]. Originally, it was obtained under commutativity assumption and for Markovian operators on abstract state spaces. We remove these restrictions. We recall that $T \in \mathcal{L}(\mathfrak{X})$ is quasi-compact if $\left\|T^{n}-K\right\|<1$ for some natural $n$ and a compact operator $K \in \mathcal{L}(\mathfrak{X})$.

Theorem 2.6 Let $T \in \mathcal{L}(\mathfrak{X})$ and $P: \mathfrak{X} \rightarrow \mathfrak{X}$ be a linear projection. If $\delta_{P}(T)<1$, $T\left(N_{P}\right) \subseteq N_{P}$, and $T P$ is a quasi-compact contraction, then $T$ is also quasi-compact.

Proof Without loss of generality, we will assume that $P \neq I$. It follows from Proposition 2.4 that $\left\|T^{n}(I-P)\right\| \rightarrow 0$ and by Theorem $2.2, \delta_{P}\left(T^{n}\right) \leq \delta_{P}(T)^{n} \rightarrow 0$. Let $\varepsilon>$ 0 be fixed. We choose $m \in \mathbb{N}$, such that $\frac{\delta_{P}(T)^{m+1}}{1-\delta_{P}(T)}<\frac{\varepsilon}{4(1+\|P\|)}$. By quasi-compactness of $T P$, there exists natural $i \geq m$, such that $\left\|(T P)^{i}-K_{i}\right\|=\gamma<1$, where $K_{i} \in \mathcal{L}(\mathfrak{X})$ is compact. We notice that $\left\|(T P)^{n i}-K_{n i}\right\| \leq \gamma^{n}$ for some compact $K_{n i} \in \mathcal{L}(\mathfrak{X})$. Let $n_{\varepsilon} \in \mathbb{N}$ be such that $\left\|(T P)^{n_{\varepsilon} i-l}-K_{n_{\varepsilon} i-l}\right\| \leq \gamma^{n_{\varepsilon}-m}<\frac{\varepsilon}{4(m+1)\|I-P\| \cdot \max \left\{1,\|T\|^{m}\right\}}$ for all $l=0,1, \ldots, m$, where $K_{n_{\varepsilon} i-l} \in \mathcal{L}(\mathfrak{X})$ are compact.

By the mathematical induction, for every natural $n$, we have the representation

$$
T^{n}=T^{n}(I-P)+T^{n} P=(T P)^{n}+\sum_{j=1}^{n} T^{j}(I-P)(T P)^{n-j} .
$$


We notice that

$$
\begin{aligned}
\left\|\sum_{l=m+1}^{n} T^{l}(I-P)(T P)^{n-l}\right\| & \leq \sum_{l=m+1}^{n}\left\|T^{l}(I-P)(T P)^{n-l}\right\| \\
& \leq \sum_{l=m+1}^{\infty}\left\|T^{l}(I-P)\right\| \\
& \leq(1+\|P\|) \sum_{l=m+1}^{\infty} \delta_{P}\left(T^{l}\right) \\
& \leq(1+\|P\|) \frac{\delta_{P}(T)^{m+1}}{1-\delta_{P}(T)}<\frac{\varepsilon}{4} .
\end{aligned}
$$

Now, estimate

$$
\begin{aligned}
& \left\|T^{n_{\varepsilon} i}-K_{n_{\varepsilon} i}-\sum_{l=1}^{m} T^{l}(I-P) K_{n_{\varepsilon} i-l}\right\| \\
& =\left\|(T P)^{n_{\varepsilon} i}-K_{n_{\varepsilon} i}+\sum_{l=1}^{n_{\varepsilon} i} T^{l}(I-P)(T P)^{n_{\varepsilon} i-l}-\sum_{l=1}^{m} T^{l}(I-P) K_{n_{\varepsilon} i-l}\right\| \\
& =\|(T P)^{n_{\varepsilon} i}-K_{n_{\varepsilon} i}+\sum_{l=1}^{m} T^{l}(I-P)(T P)^{n_{\varepsilon} i-l}-\sum_{l=1}^{m} T^{l}(I-P) K_{n_{\varepsilon} i-l} \\
& \quad+\sum_{l=m+1}^{n_{\varepsilon} i} T^{l}(I-P)(T P)^{n_{\varepsilon} i-l} \| \\
& \leq\left\|(T P)^{n_{\varepsilon} i}-K_{n_{\varepsilon} i}\right\|+\sum_{l=1}^{m}\left\|T^{l}(I-P)\right\|\left\|(T P)^{n_{\varepsilon} i-l}-K_{n_{\varepsilon} i-l}\right\| \\
& \quad+\left\|\sum_{l=m+1}^{n_{\varepsilon} i} T^{l}(I-P)(T P)^{n_{\varepsilon} i-l}\right\| \\
& <\frac{\max \left\{1,\|T\|^{m}\right\} \cdot\|I-P\| \varepsilon}{4(m+1)\|I-P\| \cdot \max \left\{1,\|T\|^{m}\right\}}+\frac{\varepsilon}{4}<\varepsilon .
\end{aligned}
$$

The operators $K_{n_{\varepsilon} i}+\sum_{l=1}^{m} T^{l}(I-P) K_{n_{\varepsilon} i-l}$ are compact, so $T$ is quasi-compact.

We end the section showing some relations between the projections $P$ and $I-P$ (cf. [27, Proposition 3.1]).

Corollary 2.7 Let $T \in \mathcal{L}(\mathfrak{X})$ satisfy $\left\|T^{n}\right\| \geq \varepsilon>0$ for all $n \in \mathbb{N}$ (for instance, $T$ is Markovian) and $P$ be a linear projection. If $T\left(N_{P}\right) \subseteq N_{P}$ and $T\left(N_{I-P}\right) \subseteq N_{I-P}$, then at most one of the statements below holds:

(1) $\delta_{P}\left(T^{n}\right)<1$ for some $n \in \mathbb{N}$,

(2) $\delta_{I-P}\left(T^{n}\right)<1$ for some $n \in \mathbb{N}$. 
Proof If both conditions hold, then by Proposition 2.4, we get

$$
\left\|T^{n}(I-P)\right\| \rightarrow 0 ;\left\|T^{n} P\right\| \rightarrow 0 .
$$

It follows that:

$$
\left\|T^{n}\right\| \leq\left\|T^{n}(I-P)\right\|+\left\|T^{n} P\right\| \rightarrow 0
$$

contradicting that the norms $\left\|T^{n}\right\|$ are separated from 0 .

\section{Uniform Ergodicity}

This section is devoted to convergence of iterates $T^{n}$, obtained with the use of Dobrushin's ergodicity coefficients.

Definition 3.1 Let $P$ be a linear projection on the Banach space $\mathfrak{X}$. We say that $T \in$ $\mathcal{L}(X)$ is uniformly $P$-ergodic, if $\left\|T^{n}-P\right\| \rightarrow 0$.

Theorem 3.2 Let $T \in \mathcal{L}(X)$ and $P \neq I$ be a projection. Then, $T$ is uniformly $P$ ergodic if and only if that $T\left(N_{P}\right) \subseteq N_{P}, T P=P$ (equivalently, $T P=P T=P$ ), and $\delta_{P}\left(T^{k}\right)<1$ for some $k \in \mathbb{N}$.

Proof Let $T$ be a uniformly $P$-ergodic operator. Applying Proposition 2.4, we get:

$$
\delta_{P}\left(T^{n}\right)=\sup _{x \in N_{P}, x \neq 0} \frac{\left\|T^{n} x\right\|}{\|x\|}=\sup _{x \in N_{P}, x \neq 0} \frac{\left\|T^{n} x-P x\right\|}{\|x\|} \leq\left\|T^{n}-P\right\| \rightarrow 0,
$$

so $\delta_{P}\left(T^{k}\right)<1$ for some $k \in \mathbb{N}$.

Moreover, from

$$
\left\|T^{n+1}-T P\right\|=\left\|T\left(T^{n}-P\right)\right\| \rightarrow 0 \text { and }\left\|T^{n+1}-P T\right\|=\left\|\left(T^{n}-P\right) T\right\| \rightarrow 0,
$$

we get commutativity $P=T P$ and $P=P T$. In particular, $T\left(N_{P}\right) \subseteq N_{P}$.

In the opposite direction, we apply our Proposition 2.2 and get $\left\|T^{n}(I-P)\right\| \rightarrow 0$, so $\left\|T^{n}-P\right\|=\left\|T^{n}-T^{n} P\right\| \rightarrow 0$.

Remark 3.3 The case when $T$ is uniformly $I$-ergodic is trivial. Simply $P=I$, and then, $\delta_{I}\left(T^{k}\right)<1$ does not occur.

Combining the above theorem with the Proposition 2.3, we get

Corollary 3.4 If $T$ is uniformly $P$-ergodic and $\delta_{P}(T)=0$, then $T=P$.

The uniform $P$-ergodicity is quite a strong property. We shall also consider its weaker alternative discussing the asymptotic behavior of $\left\|T^{n} x-T^{n} y\right\|$. Expressing it in Dobrushin's language, we propose to study the limit of $\delta_{P}\left(T^{n}\right)$ (for contractions, such a limit does exist). 
Definition 3.5 Let $P$ be a linear projection on $\mathfrak{X}$. We say that a linear contraction $T \in \mathcal{L}(\mathfrak{X})$ is weakly $P$-ergodic, if $\delta_{P}\left(T^{n}\right) \rightarrow 0$.

Now, we will discuss, from the perspective of Dobrushin's ergodicity notion, the geometry of selected semigroups. We start from general sets, and finally, we will arrive to abstract "Markovian" semigroups. Given a linear projection $P \in \mathcal{L}(\mathfrak{X})$, which is assumed to be a contraction, we define

$$
\begin{aligned}
& \mathcal{L}_{r}^{u}(P):=\{T \in \mathcal{L}(\mathfrak{X}): T \text { is uniformly } P-\text { ergodic and }\|T\| \leq r\}, \\
& \mathcal{L}_{r}^{w}(P):=\{T \in \mathcal{L}(\mathfrak{X}): T \text { is weakly } P-\text { ergodic and }\|T\| \leq r\}, \\
& \mathcal{L}_{r}^{E}(P):=\{T \in \mathcal{L}(\mathfrak{X}): P T=T P=P,\|T\| \leq r\}, \\
& \mathcal{L}_{r}^{\text {com }}(P):=\{T \in \mathcal{L}(\mathfrak{X}): P T=T P,\|T\| \leq r\} .
\end{aligned}
$$

The following inclusions are obvious: $\mathcal{L}_{r}^{u}(\mathfrak{X}) \subset \mathcal{L}_{r}^{w}(\mathfrak{X}), \mathcal{L}_{r}^{u}(\mathfrak{X}) \subset \mathcal{L}_{r}^{E}(\mathfrak{X})$ and $\mathcal{L}_{r}^{w}(\mathfrak{X}) \cap \mathcal{L}_{r}^{E}(\mathfrak{X})=\mathcal{L}_{r}^{u}(\mathfrak{X})$.

Moreover, the second inclusion is in some sense optimal, as we explain below.

Theorem 3.6 With the notation as above, the following inclusions hold:

(1) $\mathcal{L}_{1}^{u}(P)$ is a norm-open and dense subset of $\mathcal{L}_{1}^{E}(P)$.

(2) $\mathcal{L}_{1}^{w}(P) \cap \mathcal{L}_{1}^{\text {com }}(P)$ is a norm-open and dense subset of $\mathcal{L}_{1}^{\text {com }}(P)$.

Proof We notice that $\mathcal{L}_{1}^{\text {com }}(P), \mathcal{L}_{1}^{E}(P)$ are norm-closed subsets of the closed unit ball $\mathcal{L}_{1}(\mathfrak{X})$. Let $T \in \mathcal{L}_{1}^{E}(P)$ and $0<\varepsilon<1$ be fixed. We set

$$
T^{(\varepsilon)}:=\left(1-\frac{\varepsilon}{2}\right) T+\frac{\varepsilon}{2} P
$$

Clearly, $T^{(\varepsilon)} \in \mathcal{L}_{1}^{E}(\mathfrak{X}),\left\|T^{(\varepsilon)}-T\right\| \leq \varepsilon,\left\|T^{(\varepsilon)}\right\| \leq 1$ as both $T$ and $P$ are contractions $\left(\|T\|=\|P\|=1\right.$ actually, as long as $P$ is non-zero). If $x \in N_{P}$, then

$$
\frac{\left\|T^{(\varepsilon)} x\right\|}{\|x\|}=\left(1-\frac{\varepsilon}{2}\right) \frac{\|T x\|}{\|x\|} .
$$

Taking supremum over all such $x$, we get $\delta_{P}\left(T^{(\varepsilon)}\right)=\left(1-\frac{\varepsilon}{2}\right) \delta_{P}(T)$. Therefore, $\delta_{P}\left(T^{(\varepsilon)}\right)<1$, so applying the Theorem 3.2, we obtain that $T^{(\varepsilon)}$ is uniformly $P$ ergodic. $\mathcal{L}_{1}^{u}(P)$ is a norm-dense subset of $\mathcal{L}_{1}^{E}(P)$.

Concerning openness, for $n \in \mathbb{N}$, consider sets

$$
U_{n}=\left\{T \in \mathcal{L}_{1}(\mathfrak{X}): P T=T P=P ; \delta_{P}\left(T^{n}\right)<1\right\} .
$$

Clearly, $\mathcal{L}_{1}^{u}(P)=\bigcup_{n \in \mathbb{N}} U_{n}$, so it is enough to prove that $U_{n}$ are norm-open in $\mathcal{L}_{1}^{E}(P)$. If $T \in U_{n}$ is fixed, then for every $H \in \mathcal{L}_{1}^{E}(P)$ with $\|T-H\|<\frac{1-\delta_{P}\left(T^{n}\right)}{2 n}$, we have (apply Theorem 2.2)

$$
\begin{aligned}
& \left|\delta_{P}\left(T^{n}\right)-\delta_{P}\left(H^{n}\right)\right| \leq\left\|T^{n}-H^{n}\right\| \leq\left\|T^{n}-T^{n-1} H\right\|+\left\|T^{n-1} H-H^{n}\right\| \\
& \leq\|T-H\|+\left\|T^{n-1}-H^{n-1}\right\| \leq n\|T-H\|<\frac{1-\delta_{P}\left(T^{n}\right)}{2} .
\end{aligned}
$$


Therefore, $\delta_{P}\left(H^{n}\right)<1, H \in U_{n}$ and the proof is completed.

To prove (2), we may copy the proof of (1) replacing $\mathcal{L}_{1}^{u}(P)$ for $\mathcal{L}_{1}^{w}(P) \cap \mathcal{L}_{1}^{\text {com }}(P)$ and $\mathcal{L}_{1}^{E}(P)$ for $\mathcal{L}_{1}^{\text {com }}(P)$.

\section{Abstract Markovian Semigroups}

Let $\xi \in \mathfrak{X}^{*}$ be a continuous (real) functional on a Banach space $(\mathfrak{X},\|\cdot\|)$ and $\mathcal{K}_{\xi} \subset \mathfrak{X}$ be a norm-closed, bounded and convex subset of $\mathfrak{X}$ (denote $M_{\xi}=\sup _{x \in \mathcal{K}_{\xi}}\|x\|$ ). Assume that $\xi(x)=1$ for all $x \in \mathcal{K}_{\xi}$. Clearly, $\mathcal{K}_{\xi}$ is separated from 0 (i.e., $\inf _{x \in \mathcal{K}_{\xi}}\|x\|=m_{\xi}>$ $0)$.

Definition 4.1 A linear bounded operator $T \in \mathcal{L}(\mathfrak{X})$ is called $\xi-$ Markovian if $T^{*} \xi=\xi$ and $T\left(\mathcal{K}_{\xi}\right) \subseteq \mathcal{K}_{\xi}$. The set of all $\xi$-Markovian operators on $\mathfrak{X}$ is denoted by $\mathcal{S}_{\xi}$.

It follows directly from the definition that $\mathcal{S}_{\xi} \subset \mathcal{L}(\mathfrak{X})$ is a semigroup, which is closed for the weak operator topology. The composition operation is continuous for the strong operator topology. Of course, each $\xi$-Markovian operator $T$ has its norm $\|T\| \geq 1$, as $T^{*} \xi=\xi \neq 0$. Clearly, the identity operator $\mathbf{I d} \in \mathcal{S}_{\xi}$.

It is easy to verify that if $\mathfrak{X}=L^{1}(\mu)$, then the classical Markovian operators $T$ (sometimes called stochastic and then the adjoint operators $T^{*}$ are called Markovian) coincide with the just introduced semigroup $\mathcal{S}_{\mathbf{1}}$, where $\mathbf{1} \in L^{\infty}(\mu)$ denotes the function equal $1 \mu$ a.e. From this perspective general $\xi$-Markovian operators mimic some features of classical Markovian operators on $L^{1}(\mu)$. It is worth mentioning that in the literature, several different extensions of Markov operators are presented (cf. [1,5,6, 8,10,17,24,27,32,35]).

Repeating Example 2.5, we set $P_{y_{*}}=\xi \otimes y_{*}=\langle\cdot, \xi\rangle y_{*}$, where $y_{*} \in \mathcal{K}_{\xi}$. Clearly, $P_{y_{*}} \in \mathcal{S}_{\xi}$ is a $\xi$-Markovian projection. Moreover, $P_{y_{*}} T=P_{y_{*}}$ for every $T \in \mathcal{S}_{\xi}$. $P_{y_{*}}(u)=0$ if and only if $\xi(u)=0$. Hence, $N_{P_{y_{*}}}=\operatorname{ker} \xi=\operatorname{ker} P_{y_{*}}$. Now, for arbitrary $T \in \mathcal{S}_{\xi}$ and all $u \in N_{P_{y_{*}}}$, we have $\xi(T u)=T^{*}(\xi)(u)=\xi(u)=0$. We obtain $T\left(N_{P_{y_{*}}}\right) \subseteq N_{P_{y_{*}}}$.

If $\mathbb{R}_{+} \mathcal{K}_{\xi}-\mathbb{R}_{+} \mathcal{K}_{\xi}=\mathfrak{X}$, then the cone $C_{\xi}=\left\{t x: t \geq 0\right.$ and $\left.x \in \mathcal{K}_{\xi}\right\}$ is called generating in $\mathfrak{X}$. We notice that under this assumption, $\xi(v)=0$ if and only if $v=$ $t(x-y)$, for some $t \in \mathbb{R}$ and $x, y \in \mathcal{K}_{\xi}$. Therefore, the abstract Dobrushin ergodicity coefficient, introduced in ( $\star$ ), coincides with the classical one. Namely, for all $T \in \mathcal{S}_{\xi}$, we have

$$
\delta(T)=\sup \left\{\frac{\|T(x-y)\|}{\|x-y\|}: x, y \in \mathcal{K}_{\xi}\right\}=\sup \left\{\frac{\|T v\|}{\|v\|}: v \in N_{P_{y_{*}}}\right\}=\delta_{P_{y_{*}}}(T) .
$$

The rest of this section is devoted to topological (generic) characteristics of $\mathcal{S}_{\xi}$. It is well known that classical Markov operators, which are norm-mixing, form a dense and open subset of $\mathcal{S}_{\mathbf{1}}$ (see [3-5,19,20,23]). It has been recently extended to ordered Banach spaces satisfying some extra properties (see [10,14,17,24,27]). The category of abstract "norm mixing" elements in Banach algebras was studied in [31], but the 
obtained there results bring residuality (here, we deliver open denseness). We introduce the following.

Definition 4.2 We say that a $\xi$-Markovian operator $T \in \mathcal{S}_{\xi}$ is norm-mixing if there exists $x_{*} \in \mathcal{K}_{\xi}$, such that the operator $T$ is $P_{x_{*}}$-ergodic, where $P_{x_{*}} \in \mathcal{S}_{\xi}$ is onedimensional projection, i.e., $\lim _{n \rightarrow \infty}\left\|T^{n}-P_{x_{*}}\right\|=0$ (clearly $T x_{*}=x_{*}$ ). We denote the set of all norm mixing $\xi$-Markovian operators by $\mathcal{S}_{\xi}^{\text {mix }}$. If we fix $x_{*} \in \mathcal{K}_{\xi}$, then the subset $\left\{T \in \mathcal{S}_{\xi}:\left\|T^{n}-P_{x_{*}}\right\| \rightarrow 0\right\}$ is denoted by $\mathcal{S}_{\xi, x_{*}}^{\text {mix }}$. Accordingly, we define $\mathcal{S}_{\xi, x_{*}}=\left\{T \in \mathcal{S}_{\xi}: T\left(x_{*}\right)=x_{*}\right\}$.

We notice that studying mixing property, we may assume that $\|T\|=1$. In fact, by the Banach-Steinhaus theorem, $\sup _{n \geq 0}\left\|T^{n} x\right\|=\|x\|_{\text {. defines an equivalent norm }}$ on $\mathfrak{X}$, such that $T$ becomes a contraction with respect to it. Therefore, in the sequel (in the next section), we shall consider only contraction Markovian operators. However, in this section, we present a bit more general approach and show how notions mixing and the geometry of the space $(\mathfrak{X},\|\cdot\|)$ interplay.

We recall that a cone $C_{\xi}$ is $\kappa$-generating, if it is generating and each $x \in \mathfrak{X}$ has a decomposition $x=u-w$, where $u, w \in C_{\xi}$ satisfy $\|u\|+\|w\| \leq \kappa\|x\|$. Clearly, $\kappa \geq 1$ (cf. [7,36]). We remember that $\kappa=1$ if $\mathfrak{X}=L^{1}(\mu)$ or it is a predual of a Von Neumann algebra (cf. [5,15,17,24,27]).

In the rest of this section, we focus on Markovian families and study them using Dobrushin methods. We start with a few auxiliary lemmas. In the first one, we do not require that $C_{\xi}$ is generating.

Lemma 4.3 If $T \in \mathcal{S}_{\xi}^{\text {mix }}$, then there exists a unique $x_{*} \in \mathcal{K}_{\xi}$, such that

$$
\lim _{n \rightarrow \infty} \sup _{x \in \mathcal{K}_{\xi}}\left\|T^{n} x-x_{*}\right\|=0
$$

Proof If $T$ is $P_{x_{*}}$ ergodic, then $T P_{x_{*}}=P_{x_{*}}$. It follows that $T x_{*}=x_{*}$. For each $x \in \mathcal{K}_{\xi}$, we have $\xi\left(x-x_{*}\right)=\xi\left(x_{*}\right)-\xi(x)=0$. Thus, $x-x_{*} \in N_{P_{x_{*}}}$ for all $x \in \mathcal{K}_{\xi}$. By the Theorem 3.1, there exists a natural $k$, such that $\delta_{P_{x_{*}}}\left(T^{k}\right)<1$. We now estimate

$$
\begin{aligned}
\sup _{x \in \mathcal{K}_{\xi}}\left\|T^{n} x-x_{*}\right\| & =\sup _{x \in \mathcal{K}_{\xi}}\left\|T^{n} x-T^{n} x_{*}\right\|=\sup _{x \in \mathcal{K}_{\xi}}\left\|T^{n}\left(x-x_{*}\right)\right\| \\
& \leq \sup _{x \in \mathcal{K}_{\xi}}\left(\delta_{P_{x_{*}}}\left(T^{k}\right)\right)^{\left\lfloor\frac{n}{k}\right\rfloor} \cdot \max _{0 \leq j<k}\left\|T^{j} x-x_{*}\right\| \\
& \leq \operatorname{diam}\left(\mathcal{K}_{\xi}\right)\left(\delta_{P_{x_{*}}}\left(T^{k}\right)\right)^{\left\lfloor\frac{n}{k}\right\rfloor} \rightarrow 0 .
\end{aligned}
$$

Before we proceed to the main result of this section, we first explain the geometry of our cones $C_{\xi}$. We start with the following.

Lemma 4.4 If $C_{\xi}$ is generating in $(\mathfrak{X},\|\cdot\|)$, then there exists a positive constant $\alpha_{\xi}<\infty$, such that for every $u \in \mathfrak{X}$ satisfying $\xi(u)=0$ (i.e., $u \in$ ker $\xi$ )

$$
\inf \left\{\xi\left(u_{1}\right): u=u_{1}-u_{2} \text {, where } u_{1}, u_{2} \in C_{\xi}\right\} \leq \alpha_{\xi}\|u\|
$$


Proof As $C_{\xi}$ is generating, we have

$$
\bigcup_{j=1}^{\infty}\left\{s v-s w: v, w \in \mathcal{K}_{\xi}, 0 \leq s \leq j\right\}=\operatorname{ker} \xi
$$

By the Baire category theorem, there exists $j_{0} \in \mathbb{N}$, such that

$$
\operatorname{ker} \xi \cap \mathcal{K}\left(x_{0}, r\right) \subset \overline{\left\{s v-s w: v, w \in \mathcal{K}_{\xi}, 0 \leq s \leq j_{0}\right\}}\|\cdot\|
$$

We may assume that $x_{0}=s_{0} v_{0}-s_{0} w_{0}$ for some $s_{0}>0$ and $v_{0}, w_{0} \in \mathcal{K}_{\xi}$. Hence, each $u \in \operatorname{ker} \xi,\|u\| \leq 1$ belongs to

$$
u \in \overline{\left\{\left(\frac{s}{r} v+\frac{s_{0}}{r} w_{0}\right)-\left(\frac{s}{r} w+\frac{s_{0}}{r} v_{0}\right): v, w \in \mathcal{K}_{\xi}, 0 \leq s \leq j_{0}\right\}}\|\cdot\|
$$

Then, $u=u_{1}-u_{2}$, where $u_{1}, u_{2} \in C_{\xi}$ and $\left\|u_{1}-\left(\frac{s}{r} v+\frac{s_{0}}{r} w_{0}\right)\right\|$ may be as small as we wish. We get

$$
\xi\left(u_{1}\right)=\xi\left(u_{2}\right) \leq \frac{s}{r}+\frac{s_{0}}{r} \leq \frac{j_{0}+s_{0}}{r} .
$$

We set $\alpha_{\xi}=\frac{j_{0}+s_{0}}{r}+q$, where $q>0$ may be taken arbitrary (as small as we wish).

The next lemma is commonly known (see [7, Proposition 1.1.2]) but its proof is included here for the sake of completeness of the paper. Moreover, we can somehow see how the coefficient $\kappa$ depends on $\|\xi\|$ or $M_{\xi}$.

Lemma 4.5 Generating cones $C_{\xi}$ are $\kappa$-generating for some $\kappa \geq 1$.

Proof Let $x=t v-s w$ for some $t, s \geq 0$ and $v, w \in \mathcal{K}_{\xi}$, where $\|x\|=1$. Without loss of generality, we may assume that $t \geq s$. Then, $x=(t-s) v+s v-s w$ and $\|(t-s) v\|=(t-s)\|v\| \leq(t-s) M_{\xi}$. Moreover

$$
\|\xi\| \geq|\xi(x)|=|\xi((t-s) v)+s \xi(v-w)|=(t-s)
$$

Thus, $\|(t-s) v\| \leq\|\xi\| M_{\xi}$. Now

$$
\|s v-s w\| \leq\|x\|+\|(t-s) v\| \leq 1+\|\xi\| M_{\xi} .
$$

By the previous Lemma 4.4, $s v-s w=\widetilde{s} \widetilde{v}-\widetilde{s} \widetilde{w}$, where $\widetilde{v}, \widetilde{w} \in \mathcal{K}_{\xi}$ and

$$
0 \leq \widetilde{s} \leq \alpha_{\xi}\left(1+\|\xi\| M_{\xi}\right)
$$


It follows that:

$$
\begin{aligned}
x & =t v-s w=(t-s) v+\widetilde{s} \widetilde{v}-\widetilde{s} \widetilde{w} \\
& =(t-s+\widetilde{s})\left(\frac{t-s}{t-s+\widetilde{s}} v+\frac{\widetilde{s}}{t-s+\widetilde{s}}\right)-\widetilde{s} \widetilde{w} \\
& =(t-s+\widetilde{s}) \widetilde{\widetilde{v}}-\widetilde{s} \widetilde{w},
\end{aligned}
$$

where $\widetilde{\widetilde{v}}=\frac{t-s}{t-s+\widetilde{s}} v+\frac{\widetilde{s}}{t-s+\widetilde{s}} \widetilde{v} \in \mathcal{K}_{\xi}$. We obtain

$$
t-s+\widetilde{s}+\widetilde{s} \leq\|\xi\|+2 \alpha_{\xi}\left(1+\|\xi\| M_{\xi}\right)<\infty .
$$

Now

$$
\begin{aligned}
& \inf \left\{\left\|u_{1}\right\|+\left\|u_{2}\right\|: x=u_{1}-u_{2},\|x\|=1, u_{1}, u_{2} \in C_{\xi}\right\} \\
& \leq\|(t-s+\widetilde{s}) \widetilde{\widetilde{v}}\|+\|\widetilde{s} \widetilde{w}\|=(t-s+\widetilde{s})\|\widetilde{v}\|+\widetilde{s}\|\widetilde{w}\| \\
& \leq M_{\xi}(t-s+\widetilde{s}+\widetilde{s}) \leq M_{\xi}\left(\|\xi\|+2 \alpha_{\xi}\left(1+\|\xi\| M_{\xi}\right)\right)=\widetilde{\kappa}
\end{aligned}
$$

(obviously $\widetilde{\kappa} \geq 1$ as $M_{\xi}\|\xi\| \geq \sup _{v \in \mathcal{K}_{\xi}}|\xi(v)|=1$ ). We have proved that $C_{\xi}$ is $\kappa$-generating for each $\kappa>\widetilde{\kappa}$.

In the following results, we shall use already proved geometric properties of our cones $C_{\xi}$. Namely, if generating they are automatically $\kappa$-generating, and we have a piece of control on the parameter $\kappa \geq 1$. We obtain the following.

Lemma 4.6 Let $\xi \in \mathfrak{X}^{*}, \mathcal{S}_{\xi}$ be a $\xi$-Markovian semigroup and $C_{\xi}$ be a generating (so $\kappa$-generating cone for some $\kappa \geq 1$ ). Then, there exists a constant $M \geq 0$, such that $\sup \left\{\|T\|: T \in \mathcal{S}_{\xi}\right\}=M<\infty$. In particular, $\|x\|^{\bullet}=\sup \left\{\|T x\|: T \in \mathcal{S}_{\xi}\right\}$ is a norm on $\mathfrak{X}$, equivalent to $\|\cdot\|$.

Proof As before we set $m_{\xi}=\inf \left\{\|w\|: w \in \mathcal{K}_{\xi}\right\}>0$ and $M_{\xi}=\sup \{\|w\|: w \in$ $\left.\mathcal{K}_{\xi}\right\}<\infty$. Let us fix normalized $x \in \mathfrak{X}$. We have a decomposition $x=t u-s v$ for some $u, v \in \mathcal{K}_{\xi}$ and $t, s \in \mathbb{R}_{+}$, where $t \leq \frac{\kappa}{\|u\|} \leq \frac{\kappa}{m_{\xi}}$ and $s \leq \frac{\kappa}{\|v\|} \leq \frac{\kappa}{m_{\xi}}$. For an arbitrary $T \in \mathcal{S}_{\xi}$, we have

$$
\begin{aligned}
\|T x\| & \leq\|T(t u)\|+\|T(s v)\|=t\|T u\|+s\|T v\| \leq(t+s) \sup \left\{\|T w\|: w \in \mathcal{K}_{\xi}\right\} \\
& \leq(t+s) \operatorname{diam}\left(\mathcal{K}_{\xi}\right)<\infty
\end{aligned}
$$

It follows that $\sup \left\{\|T\|: T \in \mathcal{S}_{\xi}\right\} \leq \frac{2 \kappa}{m_{\xi}} \cdot \operatorname{diam}\left(\mathcal{K}_{\xi}\right)<\infty$. In particular, $\sup \{\|T\|$ : $\left.T \in \mathcal{S}_{\xi}\right\}=M<\infty$ and $\sup _{n}\left\|T^{n}\right\| \leq M$ for any $\xi$-Markovian operator $T$.

Define $\|x\|^{\bullet}=\sup \left\{\|T x\|: T \in \mathcal{S}_{\xi}\right\}$. Clearly, $\|x\| \leq\|x\|^{\bullet}$, so these norms are equivalent and $\mathcal{S}_{\xi}$ becomes a contraction semigroup with respect to $\|\cdot\|^{\bullet}$.

The operator $P_{x_{*}}$ is a two-sided zero in the semigroup $\mathcal{S}_{\xi, x_{*}}^{\operatorname{mix}}$, i.e., for all $T \in \mathcal{S}_{\xi, x_{*}}^{\text {mix }}$, we have $T P_{x_{*}}=P_{x_{*}} T=P_{x_{*}}$. In particular, $T^{n}-P_{x_{*}} \stackrel{\xi, x_{*}}{=}\left(T-P_{x_{*}}\right)^{n}$ for all $n$. 
Therefore, if for some natural $k$, we have $\left\|T^{k}-P_{x_{*}}\right\|<1$, then

$$
\lim _{n \rightarrow \infty}\left\|T^{n}-P_{x_{*}}\right\|=\lim _{n \rightarrow \infty}\left\|\left(T^{k}-P_{x_{*}}\right)^{\left\lfloor\frac{n}{k}\right\rfloor}\right\| \cdot \max _{0 \leq j<k}\left\|T^{j}-P_{x_{*}}\right\|=0
$$

(alternatively we may apply the Theorem 3.1 instead as $\delta_{P_{x_{*}}}\left(T^{k}\right)<1$ ). Thus

$$
\mathcal{S}_{\xi, x_{*}}^{\operatorname{mix}}=\bigcup_{k=1}^{\infty}\left\{T \in \mathcal{S}_{\xi, x_{*}}:\left\|T^{k}-P_{x_{*}}\right\|<1\right\}
$$

is a norm-open subset of $\mathcal{S}_{\xi, x_{*}}$. If $T$ is power bounded (for instance when $C_{\xi}$ is generating), then

$$
\lim _{n \rightarrow \infty}\left\|\left((1-\varepsilon) T+\varepsilon P_{x_{*}}\right)^{n}-P_{x_{*}}\right\|=0
$$

as $\left((1-\varepsilon) T+\varepsilon P_{x_{*}}\right)^{n}=(1-\varepsilon)^{n} T^{n}+\left(1-(1-\varepsilon)^{n}\right) P_{x_{*}}$ (we remember that $P_{x_{*}}$ is a semigroup zero). We have already proved a result which appeared in a classical version in [3, Theorem 3].

Corollary 4.7 Let $C_{\xi}$ be a generating cone in $(\mathfrak{X},\|\cdot\|)$. Then, for each fixed $x_{*} \in \mathcal{K}_{\xi}$, the set $\mathcal{S}_{\xi, x_{*}}^{\operatorname{mix}}$ is norm-open and dense in $\mathcal{S}_{\xi, x_{*}}$.

The next lemma characterizes mixing of abstract $\xi$-Markovian operators.

Lemma 4.8 Let $T \in \mathcal{S}_{\xi}$ be a Markovian operator, where the cone $C_{\xi}$ is generating (so $\kappa$-generating for some $\kappa \geq 1$ ). Then, $T$ is norm-mixing if and only if

$$
\lim _{n \rightarrow \infty} \sup _{x \in \mathcal{K}_{\xi}}\left\|T^{n} x-x_{*}\right\|=0
$$

for some (unique) $x_{*} \in \mathcal{K}_{\xi}$ (clearly $T x_{*}=x_{*}$ ).

Proof Applying the Lemma 4.3, we need only a proof in one direction. Suppose that

$$
\lim _{n \rightarrow \infty} \sup _{x \in \mathcal{K}_{\xi}}\left\|T^{n} x-x_{*}\right\|=0
$$

Clearly, $T x_{*}=x_{*} \in \mathcal{K}_{\xi}$. Thus, $\lim _{n \rightarrow \infty} \sup _{x \in \mathcal{K}_{\xi}}\left\|T^{n} x-P_{x_{*}} x\right\|=0$. If $u \in N_{P_{x_{*}}},\|u\| \leq 1$, then $u=v-w$ for some $v, w \in C_{\xi}$ with $\|v\|+\|w\| \leq \kappa$. As $u \in N_{P_{x_{*}}}$, we get $\xi(v)=\xi(w)$. Now

$$
\begin{aligned}
\left\|T^{n} u\right\| & =\left\|T^{n} v-T^{n} w\right\| \leq\left\|T^{n} v-\xi(v) x_{*}\right\|+\left\|T^{n} w-\xi(w) x_{*}\right\| \\
& =\xi(v)\left(\left\|T^{n} \frac{v}{\xi(v)}-x_{*}\right\|+\left\|T^{n} \frac{w}{\xi(w)}-x_{*}\right\|\right) .
\end{aligned}
$$


Clearly, $\frac{v}{\xi(v)} \in \mathcal{K}_{\xi}$ as $\xi\left(\frac{v}{\xi(v)}\right)=1$ (similarly $\frac{w}{\xi(w)} \in \mathcal{K}_{\xi}$ ). Estimating $\xi(v) \leq \frac{j_{0}+s_{0}}{r} M_{\xi}$, we finally obtain

$$
\lim _{n \rightarrow \infty} \sup _{u \in N_{P_{x_{*}}}\|u\|=1}\left\|T^{n} u\right\| \leq 2 \alpha_{\xi} M_{\xi} \lim _{n \rightarrow \infty} \sup _{x \in \mathcal{K}_{\xi}}\left\|T^{n} x-x_{*}\right\|=0 .
$$

To the rest, we may apply the Theorem 3.2.

The theorem presented below is the main result of this section. It generalizes the results obtained in [5, Theorem 2.4] for Markov operators acting on Schatten classes (where $\kappa=1$ ). Clearly, it also extends relevant results on the asymptotic behavior of iterates of Markov operators acting on $L^{1}(\mu)$ spaces (cf. [3], [19] and [23, Theorem $6]$ ), and it corresponds to [27, Theorems 7.1 and 7.3].

Theorem 4.9 Let $(\mathfrak{X},\|\cdot\|), \xi \in \mathfrak{X}^{*}, \mathcal{K}_{\xi}$, and $C_{\xi}$ be as before. If the cone $C_{\xi}$ is generating $(\kappa-$ generating for some $\kappa \geq 1)$ in $(\mathfrak{X},\|\cdot\|)$, then for $T \in \mathcal{S}_{\xi}$, the following are equivalent:

(i) $T \in \mathcal{S}_{\xi}^{\operatorname{mix}}$;

(ii) $\lim _{n \rightarrow \infty} \sup _{u, v \in \mathcal{K}_{\xi}}\left\|T^{n} u-T^{n} v\right\|=0$;

(iii) there exists a natural $k$, such that

$$
\sup _{u, v \in \mathcal{K}_{\xi}}\left\|T^{k} u-T^{k} v\right\|=\gamma<\frac{m_{\xi}}{\kappa} .
$$

Proof (i) $\Rightarrow$ (ii) has already been proved. (ii) $\Rightarrow$ (iii) is obvious. It remains to prove (iii) $\Rightarrow$ (i). Given $z_{*} \in \mathcal{K}_{\xi}$ and $u \in N_{P_{z_{*}}}$, with $\|u\|=1$, we find a decomposition $u=\widetilde{v}-\widetilde{w}$, where $\|\widetilde{v}\|+\|\widetilde{w}\| \leq \kappa\|u\|=\kappa, \widetilde{v}=t v, \widetilde{w}=t w, t \geq 0$ and $v, w \in \mathcal{K}_{\xi}$. Clearly, $t \leq \frac{\kappa}{m_{\xi}}$. We estimate

$$
\left\|T^{k} u\right\|=\left\|T^{k} \widetilde{v}-T^{k} \widetilde{w}\right\|=t\left\|T^{k} v-T^{k} w\right\| \leq \frac{\kappa}{m_{\xi}} \gamma=\varrho<\frac{\kappa}{m_{\xi}} \frac{m_{\xi}}{\kappa}=1 .
$$

It follows that for an arbitrary pair $v, w \in \mathcal{K}_{\xi}$, we have $\left\|T^{k} v-T^{k} w\right\| \leq \varrho\|v-w\|$. The transformation $T^{k}$ is a strict contraction on $\mathcal{K}_{\xi}$. By the Banach fixed point theorem, there exists $x_{*} \in \mathcal{K}_{\xi}$, such that $T^{k} x_{*}=x_{*}$. Notice that $\delta_{P_{*}}\left(T^{k}\right)<1$ and $T^{k} P_{x_{*}}=$ $P_{x_{*}} T^{k}=P_{x_{*}}$. Now, we may apply our Theorem 3.2 or repeating argumentation as in Corollary 4.7, we get $\lim _{n \rightarrow \infty}\left\|T^{n k}-P_{x_{*}}\right\|=0$. Finally

$$
\begin{aligned}
& \limsup _{n \rightarrow \infty} \sup _{\|x\| \leq 1}\left\|T^{n} x-P_{x_{*}} x\right\|=\limsup _{n \rightarrow \infty} \sup _{\|x\| \leq 1} \sup _{0 \leq j<k}\left\|T^{k n+j} x-\xi(x) x_{*}\right\| \\
& \quad=\limsup _{n \rightarrow \infty} \sup _{\|x\| \leq 1} \sup _{0 \leq j<k}\left\|T^{k n}\left(T^{j} x\right)-\xi\left(T^{j} x\right) x_{*}\right\| \\
& \leq \limsup \sup _{n \rightarrow \infty}\left\|T^{k n}(y)-\xi(y) x_{*}\right\|=0,
\end{aligned}
$$

as $\left\|T^{j}\right\| \leq M$ for all $j$ (see Lemma 4.6). We have proved that $T \in \mathcal{S}_{\xi}^{\operatorname{mix}}$. 
The following corollary easily follows from the last theorem. Its classical counterpart may be found in [3,5] and [20, Theorem 3].

Corollary 4.10 If $C_{\xi}$ is generating ( $\kappa$-generating for some $\kappa \geq 1$ ), then $\mathcal{S}_{\xi}^{\operatorname{mix}}$ is a norm-open and dense subset of $\mathcal{S}_{\xi}$.

Proof From Theorem 4.9(iii), we easily obtain that $\mathcal{S}_{\xi}^{\text {mix }}$ is norm-open in $\mathcal{S}_{\xi}$. Concerning denseness let us fix $z_{*} \in \mathcal{K}_{\xi}$ and $\varepsilon>0$. Given an arbitrary $T \in \mathcal{S}_{\xi}$, define $T_{\varepsilon}=(1-\varepsilon) T+\varepsilon P_{z_{*}} \in \mathcal{S}_{\xi}$. Let us endow the Banach space with the norm $\|\cdot\|^{\bullet}$, defined before. With the metric $\varrho(v, w)=\|v-w\|^{\bullet}$, the set $\mathcal{K}_{\xi}$ is a complete metric space and the "discrete time" semigroup $\left\{T_{\varepsilon}^{n}: n \geq 0\right\}$ acts on $\mathcal{K}_{\xi}$ as affine contractions. Hence, for any pair $v, w \in \mathcal{K}_{\xi}$, we have

$$
\left\|T_{\varepsilon}(v)-T_{\varepsilon}(w)\right\|^{\bullet} \leq(1-\varepsilon)\|v-w\|^{\bullet} .
$$

By the Banach fixed point theorem, there exists a unique $x_{*} \in \mathcal{K}_{\xi}$, such that $T_{\varepsilon} x_{*}=x_{*}$ and

$$
\sup _{u \in \mathcal{K}_{\xi}}\left\|T_{\varepsilon}^{n}(u)-x_{*}\right\|^{\bullet} \leq \text { const }_{1}(1-\varepsilon)^{n}
$$

Clearly

$$
\sup _{u \in \mathcal{K}_{\xi}}\left\|T_{\varepsilon}^{n}(u)-x_{*}\right\| \leq \text { const }_{2}(1-\varepsilon)^{n},
$$

as the norms $\|\cdot\|$ and $\|\cdot\|^{\bullet}$ are equivalent. From Lemma 4.8, the operator $T_{\varepsilon} \in \mathcal{S}_{\xi}^{\text {mix }}$. Applying Lemma 4.6 once again, we have $\left\|T-T_{\varepsilon}\right\| \leq 2 M \varepsilon$. The set $\mathcal{S}_{\xi}^{\text {mix }}$ is operator norm-dense in $\mathcal{S}_{\xi}$

We end this section giving some comments on the role of order structures on abstract Banach spaces. It is well known (see [17, Proposition A.1] and [27, Examples 2.3 and 2.4]) that every Banach space $(\mathfrak{X},\|\cdot\|)$ may be isometrically embedded into an ordered Banach space $\mathfrak{X}^{\sim}$. Simply consider $\mathfrak{X}^{\sim}=\mathfrak{X} \oplus \mathbb{R}$ with the norm $\|(x, r)\|^{\sim}=\max \{\|x\|,|r|\}$. The positive cone is defined as $\mathfrak{X}_{+}^{\sim}=\{(x, r):\|x\| \leq r\}$. It may be proved that the norm $\|\cdot\|^{\sim}$ is additive on $\mathfrak{X}_{+}^{\sim}$ and that the space $\mathfrak{X}^{\sim}$ is 1 -generating (i.e., $\mathfrak{X}^{\sim}$ is a strong abstract state space). Moreover, each contraction $T \in \mathcal{L}(\mathfrak{X})$ can be extended to $\widetilde{T} \in \mathcal{L}\left(\mathfrak{X}^{\sim}\right)$ which is a positive contraction. Namely, define $\widetilde{T}(x, r)=(T x, r)$. The dynamics of a linear contraction $T$ on $\mathfrak{X}$ is reflected in dynamics of $\widetilde{T}$. The problem emerges, when one asks about the mixing property. The convergence of Markovian $\widetilde{T}^{n}$, to a 1-dimensional projection on $\mathfrak{X}^{\sim}$, automatically forces on the limit operator $P((x, r))=(0, r)$. In particular, $\left\|T^{n}\right\| \rightarrow 0$ on the original space $\mathfrak{X}$. Otherwise, we would have $P((x, r))=\left(\lim _{n \rightarrow \infty} T^{n} x, r\right)$. Hence, $\left(x_{*}, 0\right),(0,1) \in \mathfrak{X}^{\sim}$ would be two non-zero $\widetilde{T}$-invariant vectors, contradicting normmixing. We conclude that studying mixing of a linear contraction $T$ on an abstract Banach space is as hard as studying uniform $P$-ergodicity of its extension $\widetilde{T}$ (on $\mathfrak{X}^{\sim}$ ) for 2 -dimensional projections $P$. Positivity of $\widetilde{T}$ and the order on $\mathfrak{X}^{\sim}$ will not 
help. We conclude that, when investigating the iterates $T^{n}$ of a linear contraction $T$ on an abstract Banach space $\mathfrak{X}$, we may not assume, without loss of generality, that $\mathfrak{X}$ is an abstract state space; especially when considering $P$ - norm-ergodicity for a 1-dimensional $P$.

\section{Continuous Semigroups and Dobrushin Coefficients}

It has been explained in the previous section that studying mixing of Markovian semigroups, we may actually assume that they are contractions. Therefore, in this section, we shall generally assume that $\mathcal{S}_{\xi}$ are contraction semigroups.

We will not consider Dobrushin's coefficients for one-parameter semigroups in much depth. We merely present two results indicating possibilities in this direction. A recent paper [14] is another example supporting possible developments of this topic. We begin with recalling (cf. $[9,13,18]$ ).

Proposition 5.1 Let $A \in \mathcal{L}(\mathfrak{X})$. Then

$$
T_{t}^{A}:=\sum_{n=0}^{\infty} \frac{A^{n}}{n !} t^{n}=e^{t A} \in \mathcal{L}(\mathfrak{X})
$$

form a one-parameter norm-continuous (i.e., $\mathbb{R} \ni t \rightarrow T_{t} \in \mathcal{L}(\mathfrak{X})$ is operator normcontinuous) semigroup of operators, with $A$ as its generator.

The theory of one-parameter linear semigroups is well developed. The reader is directed to $[9,13,18]$ for further explanations if necessary.

Here, we show that the convergence of $\left\{T_{t}\right\}$ may be characterized by the Dobrushin coefficient of a single element $T_{1}$. Such a property is known for classical Markovian semigroups on $L^{1}$ (see [21, Theorem 3.7] and [22, Theorem 7.4.1 and Corollary 8.7.3]). We have the following.

Theorem 5.2 Let $\left\{T_{t}\right\}$ be a uniformly (norm) continuous contraction linear semigroup on $\mathfrak{X}$, such that $T_{t} P=P$ for all $t \geq 0$, where $P$ is a linear projection (contraction) acting in a Banach space $\mathfrak{X}$. Then

(i) if $T_{1}$ is uniformly $P$-ergodic, then $\left\{T_{t}\right\}$ converges uniformly to $P$;

(ii) if $T_{1}$ is weakly $P$-ergodic, then $\delta_{P}\left(T_{t}\right) \rightarrow 0$.

Proof Without loss of generality, we shall assume that $P \neq I$. (i) follows from our Theorem 3.2. Indeed, notice first that $T_{1} P=P T_{1}=P$, and hence, Theorem 3.2 is applicable. Thus, $\delta_{P}\left(T_{1}^{k}\right)<1$ for some natural $k$. We notice that

$$
T_{\frac{1}{n}} P=\lim _{j \rightarrow \infty} T_{\frac{1}{n}} T_{1}^{j}=\lim _{j \rightarrow \infty} T_{\frac{1}{n}+j}=\lim _{j \rightarrow \infty} T_{1}^{j} T_{\frac{1}{n}}=P T_{\frac{1}{n}} .
$$


Applying Theorem 3.6 we infer that, for every fixed natural $n$, the operator $T_{\frac{1}{n}}$ is uniformly $P$-ergodic, as $\delta_{P}\left(T_{\frac{1}{n}}^{n k}\right)=\delta_{P}\left(T_{1}^{k}\right)<1$. Then

$$
\begin{aligned}
\| T_{t} & -P\|\leq\| T_{t}-T_{\lfloor t n\rfloor / n}\|+\| T_{\lfloor t n\rfloor / n}-P \| \\
& \leq\left\|T_{\lfloor t n\rfloor / n}\right\|\left\|T_{t-\lfloor t n\rfloor / n}-I\right\|+\left\|T_{\lfloor t n\rfloor / n}-P\right\| \\
& \leq\left\|T_{t-\lfloor t n\rfloor / n}-I\right\|+\left\|T_{\lfloor t n\rfloor / n}-P\right\| .
\end{aligned}
$$

Clearly, $\left\|T_{t-\lfloor t n\rfloor / n}-I\right\|$ may be as small as we wish (take $n$ large enough). For a fixed (large) $n$, the norm $\left\|T_{\lfloor t n\rfloor / n}-P\right\| \rightarrow 0$ when $t \rightarrow \infty$. Thus, (i) is proved.

To prove (ii), it is sufficient to notice

$$
\delta_{P}\left(T_{t}\right)=\delta_{P}\left(T_{t-\lfloor t\rfloor} T_{\lfloor t\rfloor}\right) \leq\left\|T_{t-\lfloor t\rfloor}\right\| \delta_{P}\left(T_{1}^{\lfloor t\rfloor}\right) \rightarrow 0 .
$$

Corollary 5.3 Let $\left\{T_{t}\right\}_{t \geq 0}$ be a uniformly (norm) continuous contraction linear semigroup on $\mathfrak{X}$, such that $T_{t} P_{x_{*}}=P_{x_{*}}$ for all $t \geq 0$, where as, before $P_{x_{*}}=\xi \otimes x_{*}$ for some $x_{*} \in \mathcal{K}_{\xi}$. Then, $\left\{T_{t}\right\}_{t \geq 0}$ is norm-mixing if and only if $T_{1}$ is $P_{x_{*}}$-ergodic.

We finish the paper with describing operators $A \in \mathcal{L}(\mathfrak{X})$ which generate uniform $P$-ergodic semigroups. We do not focus on Markovian semigroups; however, we are still restricted to contractions. We introduce the set

$$
\mathcal{C}=\left\{A \in \mathcal{L}(\mathfrak{X}):\left\{T_{t}^{A}\right\}_{t \geq 0} \text { is a contraction semigroup }\right\}
$$

Clearly, $\mathcal{C}$ is a closed subset of $\mathcal{L}(\mathfrak{X})$. The last theorem of the paper corresponds to [14, Theorem 3.8], where the considered semigroups belong to the $C_{0}$ class and their generators are generically unbounded [18, p. 15]. In particular, the topological structure of considered here sets $\mathcal{C}$ is not comparable with the one considered in [14]. We do realize that the presented below result is not surprising, but it is included for completeness of the topological structure of ergodic families of norm-continuous contraction semigroups, obtained with the use of the Dobrushin methods.

Theorem 5.4 Let $\mathfrak{X}$ be a Banach space and $P$ be a linear projection (contraction) on $\mathfrak{X}$. Then, the set

$$
\mathcal{E}_{P}=\left\{A \in \mathcal{C}:\left\{T_{t}^{A}\right\}_{t \geq 0} \text { is uniformly P-ergodic }\right\}
$$

is norm-open in

$$
\mathcal{C}_{P}=\left\{A \in \mathcal{C}: T_{1}^{A}\left(N_{P}\right) \subseteq N_{P}, T_{1}^{A} P=P\right\}
$$

Moreover, $\mathcal{E}_{P}$ is norm-dense in

$$
\mathcal{C}_{P}^{\prime}=\{A \in \mathcal{C}: A P=P A=0\} \subset \mathcal{C}_{P}
$$


Proof As before, we shall assume $\delta_{P}\left(T_{1}^{A}\right)=\gamma<1$. Clearly, $A \rightarrow \exp (A)$ is normcontinuous. Let $A \in \mathcal{E}_{P}$ be fixed. Therefore, for every $\varepsilon>0$, there exists $\varrho>0$, such that, if $\|A-B\|<\varrho, B \in C_{P}$, then $\|\exp (A)-\exp (B)\|<\varepsilon$. From Theorem 2.2(2), we have

$$
\left|\delta_{P}(\exp (A))-\delta_{P}(\exp (B))\right| \leq\|\exp (A)-\exp (B)\|
$$

Taking $\varepsilon=(1-\gamma) / 2$, we get $\delta_{P}(\exp (B))<1$. Applying our Theorem 3.2, we infer that $\mathcal{E}_{P}$ is norm-open in $\mathcal{C}_{P}$.

To prove denseness, let us fix $A \in \mathcal{C}_{P}^{\prime}$ and define

$$
A^{\varepsilon, P}:=A+\varepsilon(P-I) .
$$

Applying standard arguments (see [22, p. 236], [4, p. 50] and [23, Lemma 3]), we find the operator $A^{\varepsilon, P}$ to be a generator of a contraction semigroup $\left\{T^{\varepsilon, P}\right\}$. Notice that $A^{\varepsilon, P} \in \mathcal{C}_{P}^{\prime}$. We have a representation

$$
T_{t}^{\varepsilon, P}=\exp (-\varepsilon t)\left(T_{t}^{A}+\sum_{n=1}^{\infty} \varepsilon^{n} T_{t}^{n, P}\right),
$$

where $T_{t}^{0, P}=T_{t}^{A}$ and

$$
T_{t}^{n+1, P}=\int_{0}^{t} T_{t-s}^{A} P T_{s}^{n, P} d s .
$$

By mathematical induction, we estimate $\left\|T_{t}^{n+1, P}\right\| \leq t^{n} / n$ !, so $\left\|T_{t}^{\mathcal{E}, P}\right\| \leq 1$ (cf. [4]). Now, by Theorem 22, we get

$$
\begin{aligned}
\delta_{P}\left(T_{1}^{\varepsilon, P}\right) & \leq \exp (-\varepsilon)\left(\delta_{P}\left(T_{1}^{A}\right)+\sum_{n=1}^{\infty} \varepsilon^{n}\left\|T_{1}^{n, P}\right\|\right) \\
& \leq \exp (-\varepsilon)\left(\delta_{P}\left(T_{1}^{A}\right)+\sum_{n=1}^{\infty} \frac{\varepsilon^{n}}{n !}\right)<1 .
\end{aligned}
$$

It follows that $\left\{T^{\varepsilon, P}\right\}$ is uniformly $P$-ergodic, i.e., $A^{\varepsilon, P} \in \mathcal{E}_{P}$.

Open Access This article is licensed under a Creative Commons Attribution 4.0 International License, which permits use, sharing, adaptation, distribution and reproduction in any medium or format, as long as you give appropriate credit to the original author(s) and the source, provide a link to the Creative Commons licence, and indicate if changes were made. The images or other third party material in this article are included in the article's Creative Commons licence, unless indicated otherwise in a credit line to the material. If material is not included in the article's Creative Commons licence and your intended use is not permitted by statutory regulation or exceeds the permitted use, you will need to obtain permission directly from the copyright holder. To view a copy of this licence, visit http://creativecommons.org/licenses/by/4.0/. 


\section{References}

1. Ayupov, Sh.A.: Ergodic theorems for Markov chains on $\mathrm{O}^{*}$-algebras, (Russian) Dokl. Akad. Nauk UzSSR 7, 11-13 (1978)

2. Bardet, I.: Quantum extensions of dynamical systems and Markov semigroups, arXiv:1509.04849, (2015)

3. Bartoszek, W.: Norm residuality of ergodic operators. Bull. Polish Acad. Sci. Math. 29, 165-167 (1981)

4. Bartoszek, W.: One-parameter positive contraction semigroups are convergent. Univ. Iagel. Acta Math. 33, 49-57 (1996)

5. Bartoszek, W., Kuna, B.: On residualities in the set of Markov operators on $\mathcal{C}_{1}$. Proc. Amer. Math. Soc. 133, 2119-2129 (2005)

6. Bartoszek, K., Bartoszek, W.: A Noether theorem for stochastic operators on Schatten classes. J. Math. Anal. Appl. 452, 1395-1412 (2017)

7. Batty, C.J.K., Robinson, D.W.: Positive one-parameter semigroups on ordered Banach spaces. Acta Applicandae Mathematicae 1, 221-296 (1984)

8. Berdikulov, M.: Markov processes on order-unit spaces. Theory Probab. Appl. 53(1), 136-144 (2009)

9. Bratelli, O., Robinson, D.W.: Operator Algebras and Quantum Statistical Mechanics 1. Springer (1979)

10. Carbone, R., Sasso, E., and Umanita, V.: Structure of generic quantum Markov semigroup, Infin. Dimens. Anal. Quantum Probab. Relat. Top. 20.2 , (19 pages), (2017)

11. Carbone, R., and Jenčová, A.: On period, cycles and fixed points of quantum channel, Annales Henri Poincaré 21.1 Springer (2020)

12. Dobrushin, R.L.: Central limit theorem for nonstationary Markov chains. Theor. Probab. Appl. 1(6580), 329-383 (1956)

13. Eisner, T., Farkas, B., Haase, M., Nagel, R.: Operator theoretic aspects of Ergodic Theory. Springer (2015)

14. Erkursun-Özcan, N., Mukhamedov, F.: Stability estimates of Markov semigroups on abstract states spaces. Mediterranean J. Math. 17(2), 1-25 (2020)

15. Emel'yanov, E.Y., Wolff, M.: Positive operators on Banach spaces ordered by strongly normal cones. Positivity 7(1-2), 3-22 (2003)

16. Gaubert, S., Quo, Z.: Dobrushin's ergodicity coefficient for Markov operators on cones. Integral Equ. Oper. Theory81(1), 127-150 (2015)

17. Glück, J., Wolff, M.P.H.: Long-term analysis of positive operator semigroups via asymptotic domination. Positivity 23(5), 1113-1146 (2019)

18. Goldstein, J.A.: Semigroups of Operators and Applications. Oxford University Press (1985)

19. Iwanik, A.: Baire category of mixing for stochastic operators. Rend. Circ. Mat. Palermo (2) Suppl. 28, 201-217 (1992)

20. Iwanik, A., Rebowski, R.: Structure of mixing and category of complete mixing for stochastic operators. Ann. Polonici Mathematici. 56, 3 (1992)

21. Lasota, A.: Invariant principle for discrete time dynamical systems. Univ. Iagel. Acta Math. 31, 111-127 (1994)

22. Lasota, A., Mackey, M.C.: Chaos, Fractals and Noise: Stochastic Aspects of Dynamics. Springer, New York (1993)

23. Lasota, A., Myjak, J.: Generic properties of stochastic semigroups. Bull. Pol. Ac. Sci. Mat. 40(4), 283-292 (1992)

24. Makarov, B.M., and Weber, M.R.: On the asymptotic behaviour of some positive semigroups, arXiv:1901.04382v1 (2019)

25. Mukhamedov, F.: On $L^{1}$-weak ergodicity of nonhomogeneous discrete Markov processes and its applications. Revista Matemática Complutense 26(2), 799-813 (2013)

26. Mukhamedov, F.: Uniform stability and weak ergodicity of nonhomogeneous Markov chains defined on ordered Banach spaces with a base. Positivity 20, 135-153 (2016)

27. Mukhamedov, F., Al-Rawashdeh, A.: Generalized Dobrushin ergodicity coefficient and uniform ergodicities of Markov operators. Positivity 24(4), 855-890 (2020)

28. Mukhamedov, F., and Al-Rawashdeh, A.: Generalized Dobrushin ergodicity coefficient and ergodicities of non-homogeneous Markov chains, arXiv:2001.07703 (2020)

29. Mukhamedov, F., Temir, S., Akin, H.: On mixing and completely mixing properties of positive $L^{1}-$ contractions of finite von Neumann algebras. Proc. Amer. Math. Soc. 134(3), 843-850 (2005) 
30. Mukhamedov, F., Temir, S., Akin, H.: On stability properties of positive contractions of $L^{1}$-spaces associated with finite von Neumann algebras. Coll. Math. 105, 259-269 (2006)

31. Rudnicki, R.: Generic properties of multiplicative functions and stochastic semigroups. Bull. Pol. Ac. Sci. Mat. 45(1), 7-16 (1997)

32. Sarymsakov, T.A., Zimakov, N.P.: Ergodic principle for the Markov semigroup in ordered normal spaces with basis (in Russian). Dokl. Akad. Nauk SSSR 289(3), 554-558 (1986)

33. Seneta, E.: On the historical development of the theory of finite inhomogeneous Markov chains, Mathematical Proceedings of the Cambridge Philosophical Society 74.3 Cambridge University Press (1973)

34. Størmer, E.: Positive Linear Maps of Operator Algebras. Springer, Berlin (2013)

35. Szehr, O., Wolf, M.M.: Perturbation bounds for quantum Markov processes and their fixed points. J. Math. Phys. 54, 10 (2013)

36. Yost, D.: A base norm space whose cone is not 1-generating. Glasgow Math. J. 25, 35-36 (1984)

Publisher's Note Springer Nature remains neutral with regard to jurisdictional claims in published maps and institutional affiliations. 